Textures and Microstructures, 1988, Vols. 8 \& 9, pp. 601-610

Reprints available directly from the publisher

Photocopying permitted by license only

(C) 1988 Gordon and Breach Science Publishers Inc.

Printed in the United Kingdom

\title{
Plastic Anisotropy of Titanium, Zirconium and Zircaloy 4 Thin Sheets
}

\author{
P. VEDOYA, A. POCHETTINO and R. PENELLE*
}

Departamento Materiales, CNEA, Av. Libertador 8250, 1429 Buenos Aires,

Argentina

* Laboratoire de Métallurgie Structurale, UA CNRS 1107, Université Paris Sud, 91405 Orsay Cedex France

(Received March 19, 1987; in final form July 31, 1987)

\section{Dedicated to the memory of Professor Günter Wassermann}

Experimental evolution of Lankford anisotropy coefficient $R(\alpha)$ versus the amount of strain in tension $E_{11}$ and versus the angle $\alpha$ between rolling direction and the tensile test direction for Titanium, Zirconium $(\mathrm{Zr} 702)$ and Zircaloy $4(\mathrm{Zr} 704)$ thin sheets points out that $R(\alpha)$ is not constant with deformation; indeed $R(\alpha)$ tends to infinity when $\alpha>45^{\circ}$ and $E_{11}<0.02$ which corresponds to a planar deformation state. This behaviour can be interpreted by using a model of Sachs type for tensile tests performed along either the rolling direction or the transverse direction by considering only prismatic slip.

KEY WORDS: Lankford parameter, Titanium, Zirconium, Zircaloy, ODF analysis, Sachs model, prism slip.

\section{INTRODUCTION}

Textured hexagonal materials such as titanium, zirconium and their alloys present a strong plastic anisotropy. Several works after those of Thornburg and Piehler (1975) have been devoted to plastic behaviour simulation of these materials based on Taylor model (Dervin, 1978, Pochettino et al., 1981, Carre, 1984). In its classical form, Taylor model requires five independent slip systems or a combination of slip and twinning to accommodate any given 
deformation; the previous authors used a combination of two prismatic slip systems, plus two slip systems which are either the basal $\{0001\}\langle 11 \overline{2} 0\rangle$ or a combination basal-pyramidal $\{10 \overline{1} 1\}\langle 11 \overline{2} 0\rangle$ and one twinning system to accommodate deformation along [0001] axis. Selection of basal and pyramidal slips is related to their respective critical resolved shear stress CRSS; for titanium CRSS values were taken from experimental results due to Naka (1983), obtained on single crystals where only prismatic and basal slips were observed. Twinning systems commonly observed are respectively $\{1 \overline{1} 2\}\langle 11 \overline{2} 3\rangle$ and $\{10 \overline{1} 2\}\langle 10 \overline{1} 1\rangle$ for compression and extension along $c$ axis. Tomé and Kocks (1985) used a more generalized analytical solution to select the combination of prismatic, pyramidal $(c+a)$ slip and also $\{10 \overline{1} 2\}\langle 10 \overline{1} 1\rangle$ twinning active system in tension.

Theoretical description of anisotropic plastic behaviour of textured polycrystalline sheets under planar stress state is provided by knowledge of yield loci. Unfortunately, only a reduced number of experimental points and tangents to yield loci can be determined in order to verify the validity of simulation. Among these experimental data, the Lankford coefficient $R(\alpha)$, which is related to the deep drawing ability, can be used:

$$
R(\alpha)=d E_{22} / d E_{33}
$$

where $\alpha$ is the angle between the rolling and the tensile test directions and $E_{11}, E_{22}, E_{33}$ the longitudinal, transverse and normal deformations in a tensile test.

In the present work, experimental results point out that $R(\alpha)$ is not constant with amount of strain for Titanium, Zirconium and Zircaloy 4 thin sheets. Indeed $R(\alpha)$ tends to the infinity when $\alpha>45^{\circ}$ and $E_{11}$ tends to zero, what corresponds to a planar deformation state.

In order to explain these results, a study of the microstructure of the deformed state was performed. Slip and twinning active systems were analyzed by means of optical and transmission electron microscopy for samples deformed along rolling (R.D.) and transverse (T.D.) directions (Pochettino et al., to be published). From microstructural observations it has been pointed out that only prismatic slip $\{10 \overline{1} 0\}\langle 11 \overline{2} 0\rangle$ is occurring at low amount of strain, according to Naka (1983). For tensile tests along R.D. twinning is 
not practically observed for strain amounts $E_{11}<0.06$, however when tests are performed along T.D. twinning is detected from the onset of plastification, but twinned volume fraction remains less than 5\%. Besides, voids at grain boundaries involving discontinuity of deformation have been observed Da Costa et al. (1986). That is the reason for which a Sachs type model has been used to simulate plastic deformation of Zirconium, Zircaloy 4 and Titanium for low deformations. By using this model with two prismatic slip systems and taking into account the crystallographic texture, it has been possible to predict semi-quantitatively evolution of $R$ with $\alpha$ and with the amount of deformation in tension.

\section{EXPERIMENTAL RESULTS}

For all sheets at recrystallized state the Lankford coefficient was determined from ISO 50 samples cut at different values of $\alpha$ between the rolling and the transverse directions by using either two extensometers for dynamic measurements or a micrometer for static determinations, strain rate $\dot{\varepsilon}$ was equal to $10^{-4} \mathrm{~s}^{-1}$.

In order to give a clearer representation of the Eq. (1), the Lankford coefficient for a given angle $\alpha$ was obtained as the tangent to the $E_{22}$ versus $E_{33}$ curve, Figure 1. In this figure, each point of the curve is associated to an increment of deformation $\Delta E_{11}=0.01$. Figure 1a presents experimental data obtained for titanium, curves for $\alpha=30^{\circ}$ and $60^{\circ}$ are not drawn, but they have a very similar shape to those corresponding to $\alpha=45^{\circ}$ and $90^{\circ}$ respectively, as it can be noticed from the Table 1 . An important result can be obtained from the Figure 1a, for $\alpha<45^{\circ}, R(\alpha)$ is nearly constant at any deformation $E_{11}$, whereas beyond $\alpha=45^{\circ}$ the Lankford coefficient presents an anomaly, it is constant and has a finite value for $E_{11} \geqslant E_{11}^{0}$ but it tends to the infinity when $E_{11}$ tends to zero. In this case, the onset of plastification is near a planar strain state. Experimental data of Figure (1) can be represented by a family of straight lines

$$
E_{22}(\alpha)=E_{22}^{0}(\alpha)+R(\alpha) \cdot E_{33}(\alpha)
$$

This equation is valid for deformations $E_{11}(\alpha)>E_{11}^{0}(\alpha)$.

This last value can be obtained by taking into account that for a 

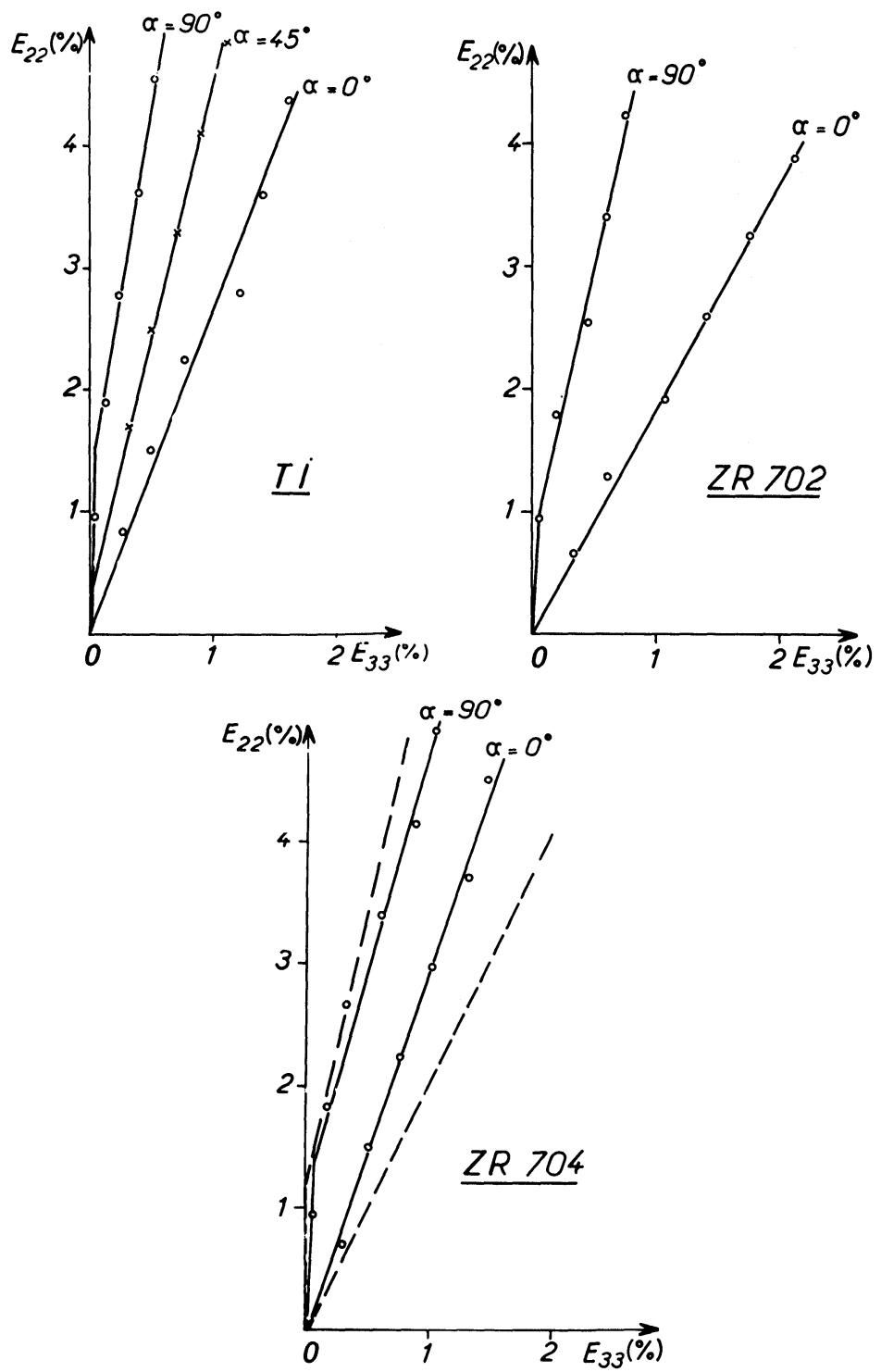

Figure 1 Experimental $E_{22}=f\left(E_{33}\right)$ curves for: Titanium, Zirconium, Zircaloy 4 sheets. The $y$ Lankford coefficient $R$ is defined as the tangent to curves in this representation, $R \simeq \infty$ for $\alpha>45^{\circ}$, when $E_{11}$ tends to zero. - - - simulated $E_{22}=f\left(E_{33}\right)$ curves. 
Table 1 Parameters of the equation $E_{22}(\alpha)=E_{22}^{0}(\alpha)+$ $R(\alpha) \times E_{33}(\alpha)$

\begin{tabular}{lrll}
\hline & $\alpha^{0}$ & $E_{22}^{0}(\alpha)$ & $R(\alpha)$ \\
\hline Titanium & 0 & 0 & 2.7 \\
& 30 & 0 & 4.0 \\
& 45 & $3.0 \times 10^{-3}$ & 4.5 \\
& 60 & $1.3 \times 10^{-2}$ & 6.2 \\
Zirconium & 90 & $1.3 \times 10^{-2}$ & 6.0 \\
& 0 & 0 & 1.8 \\
Zircaloy 4 & 90 & $7.5 \times 10^{-3}$ & 4.1 \\
& 0 & 0 & 2.95 \\
& 90 & $1.15 \times 10^{-2}$ & 3.8 \\
\hline
\end{tabular}

planar strain state $\left(E_{33}(\alpha)=0\right)$, it follows that $E_{22}^{0}(\alpha)=-E_{11}^{0}(\alpha)$. Similar behaviour is observed in the case of Zirconium and Zircaloy 4. From Eq. (2) it can be also noticed that it would be dangerous to extrapolate the value of $R(\alpha)$ corresponding to $E_{11}=0$ from deformations $0.05 \leqslant E_{11}<0.2$ as it is often done for materials having bcc or fcc structure, as it was mentioned previously by Truszkowski et al. (1985).

Crystallographic textures of the samples have been determined from $\{0002\},\{10 \overline{1} 0\}$ and $\{10 \overline{1} 1\}$ pole figures obtained by $X$ ray diffraction experiments using reflexion transmission method Dervin (1978). Then, the Orientation Distribution Functions, O.D.F., of the as received material and of samples strained up to $E_{11} \simeq 0.07$ along rolling and transverse directions have been computed for an expansion order $L=16$ without taking into account the odd part of the texture function Bunge (1982). The principal components of Titanium and Zircaloy 4 sheets are shown in O.D.F. sections at $\psi=90^{\circ}$, Figure 2 . For Titanium, texture can be described by means of two main components (2115) [0110], $\left(\psi=90^{\circ}, \theta=30^{\circ}, \varphi=30^{\circ}\right)$, (1103)[1120] $\left(\psi=90^{\circ}, \theta=30^{\circ}, \varphi=0,60^{\circ}\right)$ and of a minor component (0001)[2īi 0$]\left(\psi=90^{\circ}, \theta=\varphi=0^{\circ}\right)$ where $\psi, \theta, \varphi$ are Euler angles in the Roe representation. For Zirconium and for Zircaloy 4 sheets, the principal component of the recrystallization texture (1103)[ $[\overline{1} \overline{1} 20]$ is joined with the $(\overline{2} 115)[0 \overline{1} 10], \quad(0001)[2 \overline{1} \overline{1} 0]$ orientations.

After deformation in tension along R.D. and T.D., it is observed that distribution of $\{10 \overline{1} 0\}$ planes is modified; in the case of Titanium, deformation along R.D. strengthens the $(\overline{2} 115)[0 \overline{1} 10]$ and 
turns toward the (1103)[1]120] orientation when tensile test is performed along the T.D. for Zircaloy 4 texture is slightly affected by tension along R.D. or T.D. at least qualitatively. Texture evolution of Titanium and Zircaloy 4 is mainly associated with changes in $\varphi: \psi$ and $\theta$ remaining nearly constant what corresponds to a rotation about [0001] axis. As anisotropy of plastic deformation for these materials is depending on distribution of $c$ axis, variations of $R(\alpha)$ are not to be expected though texture is evolving with deformation amount (Pochettino et al., 1984).

\section{DISCUSSION}

If induced anisotropy does not involve any variation of $R(\alpha)$ with deformation, that observed could be due to the difficulty to activate plastic deformation. A semi-quantitative explanation of results can be obtained by using a model of Sachs type, where it has been assumed that only prismatic slip contributes to plastic deformation: activation of other slip and twinning systems being neglected in this first step. The principal texture components and their dispersions in the plane N.D.-T.D. have been introduced by means of equivalent single crystals weighted by their volume fraction in the sheet, Mingolo et al. (1986).

The development of the calculation is very simple. By means of the well known relation:

$$
\sigma_{i j}=\sum_{k l} a_{i k} a_{j l} \sum_{k l}
$$

It is possible to describe the components $\sigma_{i j}$ of the stress tensor in the crystal coordinate system associated to the grains $(X / /[10 \overline{10}], Y / /[1210], Z / /[0001])$ as a function of the applied stress $\sum_{k l}$ referred to the sample system (defined by R.D.-T.D.-N.D.). The terms $a_{i j}$ are the components of the rotation matrix relating both axes systems.

In the crystal system, the calculation of the shear stress for each slip system " $s$ " can be done by using the generalised Schmid law:

$$
\tau^{s}=\sum_{l j} m_{i j}^{s} \sigma_{i j}
$$


Table 2 Inverse of the Schmid factor for prismatic single slip in the N.D.-T.D. plane for tensile tests along rolling (R.D.) and transverse (T.D.) directions

\begin{tabular}{lll}
\hline Slip system & $/ /$ & $m^{-1}$ \\
\hline$(0 \overline{1} 10)[\overline{2} 110]$ & R.D. & $\frac{3}{4}\left(\cos ^{2} \varphi-\sin ^{2} \varphi\right)-\frac{1}{2} \sin \varphi \cos \varphi$ \\
& T.D. & $\cos ^{2} \theta\left(\frac{3}{4}\left(\sin ^{2} \varphi-\cos ^{2} \varphi\right)+\frac{1}{2} \sin \varphi \cos \varphi\right)$ \\
$(10 \overline{1} 0)[1 \overline{2} 10]$ & R.D. & $-\sin \varphi \cos \varphi$ \\
& T.D. & $\cos ^{2} \theta \sin \varphi \cos \varphi$ \\
$(1 \overline{1} 00)[\overline{1} \overline{1} 20]$ & R.D. & $\frac{3}{4}\left(\sin ^{2} \varphi-\cos ^{2} \varphi\right)-\frac{1}{2} \sin \varphi \cos \varphi$ \\
& T.D. & $\cos ^{2} \theta\left(\frac{3}{4}\left(\cos ^{2} \varphi-\sin ^{2} \varphi\right)+\frac{1}{2} \sin \varphi \cos \varphi\right)$ \\
\hline
\end{tabular}

where

$$
m_{i j}^{s}=\frac{1}{2}\left(b_{i}^{s} n_{j}^{s}+b_{j}^{s} n_{i}^{s}\right)
$$

and where $b$ and $n$ are the unit vector of slip direction and of the normal to the slip plane.

The relations between the external applied stress and the shear stress $\tau^{s}$ for prismatic slip are presented in Table 2 . They correspond to the principal texture components defined by $\psi=90^{\circ}$ for any values of $\theta$ and $\varphi$.

In h.c.p. materials, prismatic slip is possible for three systems, two being only independent. It is obvious in this case that deformation along $c$ is impossible. Then, in the crystal axis, the strain tensor components non equal to zero to be considered are $\varepsilon_{x x}, \varepsilon_{y y}$ and $\varepsilon_{x y}$. For each slip system (s), a linear hardening law has been chosen:

$$
\tau^{s}=\tau_{0}+H^{s} \gamma^{s}
$$

where $\tau$ and $\tau_{0}$ are respectively the resolved and the critical resolved shear stress, $\gamma^{s}$ the shear strain amount on the system $s$ and $H^{s}$ the slope of the shear stress-shear strain curve in tension for the stage II of hcp metals. Taking into account Naka (1983) results, $\tau_{0}$ and $H^{s}$ values have been chosen such as $\tau_{0}=60 \mathrm{MPa}$ and $H^{s}=$ $200 \mathrm{MPa}$. Then, for an uniaxial external applied stress, it is possible to evaluate the quantity of shear $\gamma^{s}$ for each slip system. The equations

$$
\varepsilon_{i j}=\sum_{s} n_{i}^{s} b_{j}^{s} \gamma^{s}
$$

and

$$
E_{i j}=\sum_{k l} a_{k i} a_{l j} \varepsilon_{k l}
$$


Table 3 Deformations in the principal sample axis for $\psi=90^{\circ}$

$\delta E_{11}=-\frac{3}{4}\left(\cos ^{2} \varphi-\sin ^{2} \varphi\right)\left[\delta \gamma_{1}-\delta \gamma_{3}\right]+\frac{1}{2} \cos \varphi \cdot \sin \varphi \cdot\left[2 \delta \gamma_{2}-\delta \gamma_{1}-\delta \gamma_{3}\right]$

$\delta E_{22}=-\frac{3}{4} \cos ^{2} \theta\left(\cos ^{2} \varphi-\sin ^{2} \varphi\right)\left[\delta \gamma_{1}-\delta \gamma_{3}\right]-\frac{1}{2} \cos ^{2} \theta \cos \varphi \sin \varphi \cdot\left[2 \delta \gamma_{2}-\delta \gamma_{1}-\delta \gamma_{3}\right]$ $\delta E_{33}=-\frac{3}{4} \sin ^{2} \theta\left(\cos ^{2} \varphi-\sin ^{2} \varphi\right)\left[\delta \gamma_{1}-\delta \gamma_{3}\right]-\frac{1}{2} \sin ^{2} \theta \cos \varphi \sin \varphi \cdot\left[2 \delta \gamma_{2}-\delta \gamma_{1}-\delta \gamma_{3}\right]$

allow to know the components of the strain tensor in the crystal and the sample axes. Table 3 presents the relations between the slip amount $\gamma^{s}$ for each slip system and the resulting principal $E_{11}, E_{22}$ and $E_{33}$ deformations for the principal texture components $(\psi=$ $90^{\circ}$ ).

From Tables 2 and 3 it can be remarked that for tensile tests performed along the R.D., the Schmid factor, $m_{i j}^{s}$, is constant for grains having their $c$ axis in the N.D.-T.D. plane. Consequently, deformation by prismatic slip of grains belonging to the principal texture components is independent of the angle $\theta$ between $c$ axis and N.D., so the Lankford anisotropy coefficient is expected to be constant with deformation. For tests performed along T.D., the Schmid factor is multiplied by $\cos ^{2} \theta$. Then, when $\theta$ increases, the applied stress required to the onset of prismatic slip increases too. In this case plastic deformation is initiated in grains whose $c$ axis are near the N.D.: then, for these grains, the Lankford coefficient tends to the infinity.

By an iterative resolution of Eqs. (3) to (8) for an increasing stress it is possible to describe the resulting deformations $E_{11}, E_{22}$ and $E_{33}$ in grains. Taking into account the principal texture components, the mean value of these deformations and that of the Lankford coefficient of the polycrystalline samples have been calculated for stresses verifying the condition $\Sigma_{0} \leqslant \Sigma \leqslant 2 \Sigma_{0}$ where $\Sigma_{0}=(4 / \sqrt{3}) \tau_{0}$ is the yield stress for all the grains located in the N.D.-T.D. plane when tensile test is performed along R.D. or for the grains having their $c$ axis parallel to the N.D. when tensile test is performed along T.D. The highest value of $\Sigma$ corresponds to $E_{11}=0.07$. Figure 1 shows a good agreement between the proposed approach and experiments. For deformations $E_{11}<0.012$ along T.D., the Lankford coefficient tends to infinity; when the applied stress is sufficient to activate slip systems in grains whose $c$ axes are near the principal texture components, the anisotropy of plastic deformation decreases up to $R \simeq 4,7$. For tensile tests along R.D., a constant value of Lankford coefficient $R \simeq 2.0$ is obtained.

It must be mentioned that for tests along T.D., the term $\cos ^{2} \theta$ in 
the Schmid factor indicates that for $\Sigma=2 . \Sigma_{0}$ slip activation is only produced in grains whose orientations verify $\theta<45^{\circ}$. Nevertheless, transmission electron microscopy observations for deformations $E_{11} \simeq 0.07$, point out the activation of slip system in all the grains (Pochettino et al., to be published). It follows that slip activation in grains verifying $\theta>45^{\circ}$ might be helped by the internal stresses due to neighbouring grains which are more hardened.

The texture evolution observed can be also interpreted by this type of approach, in fact, assuming that at the beginning of deformation grains have a similar behaviour to single crystals, the well known rules of deformation geometry can be applied to estimate rotation Reid (1975). It can be easily shown, Figure 2, that

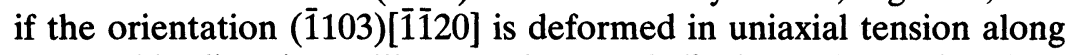
R.D., this direction will turn of around $6^{\circ}$ about the $c$ direction. This rotation will contribute to the reinforcement of the

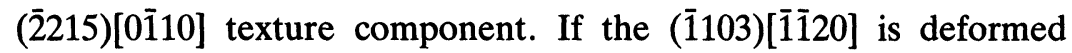
along T.D., which is in a bissector plane of two active slip directions, the $c$ axis will turn of around $2^{\circ}$ towards the (0001)[211 10 ] as it is shown in Figure 2. A similar analysis can be done for
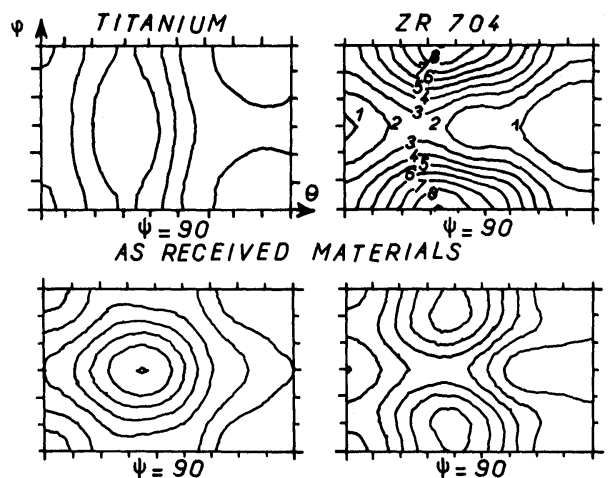

AFTER TENSILE TEST ALONG RO. (E $11 \approx 0.07)$
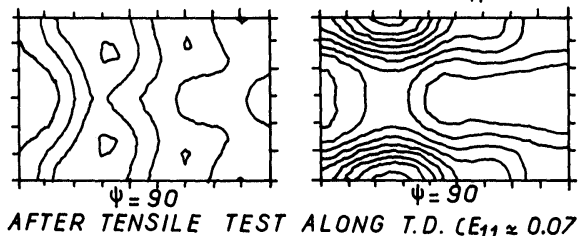

Figure 2 O.D.F. sections at $\psi=90^{\circ}$, showing texture evolution for Titanium and Zircaloy 4 after tensile tests. 
orientation ( $\overline{2} 115)[0 \overline{1} 10]$ which is stable for a deformation in tension along R.D. and instable for tension along T.D. Figure 2.

In spite of its simple assumptions, the present simulation gives correct results for deformation in tension: in a second step an intermediate model will be used, moreover, it will be taken into account dispersion of the texture, and a more realistic inter and intragranular hardening.

\section{References}

Bunge, H. J. (1982). Texture Analysis in Materials Science, Butterworths, London.

Carré, C. (1984). Thèse 3 ème Cycle, U.P.S., Orsay.

Da Costa, A. R., Pernot, M., Servant, C. and Penelle, R. (1986). Scripta Met 20, 361-364.

Dervin, P. (1978). Thèse Dr. Ing, U.P.S., Orsay.

Mingolo, N., Pochettino, A. and Tomé, C. (1986). Primer Simposio FrancoArgentino, Ciencia de Materiales, Mar del Plata, Argentina, 63-68.

Naka, S. (1983). Thèse d'Etat, U.P.S., Orsay.

Pochettino, A., Dervin, P. and Penelle, R. (1984). Proc. ICOTOM VII, the Netherlands Soc. for Materials Science, 525-529.

Pochettino, A., Vedoya, P. and Penelle, R., to be published in 8th Riso Symposium on Metallurgy and Materials Science, 1987.

Reid, C. N. (1975). Deformation geometry for materials Scientists, Pergamon Press, London.

Thornburg, D. R. and Piehler, H. R. (1975). Met Trans. 6A, 1511-1523.

Tomé, C. and Kocks, U. F. (1985). Acta Met, 33, 603-621.

Truszkowski, W. and Kloch, J. (1985). Matériaux et Techniques, 8-9, 17-20. 